\title{
Atenção Farmacêutica: \\ fundamentação conceitual e crítica para um modelo brasileiro
}

\author{
Pharmaceutical Care: \\ conceptual and critical basis to a Brazilian model
}

Daniela Angonesi ${ }^{1}$ Gil Sevalho ${ }^{2}$

\footnotetext{
${ }^{1}$ Centro Universitário Newton Paiva. Av. Silva Lobo 1730, escaninho 312, N ova Granada. 30460-000 Belo Horizonte M G. daniangonesi@hotmail.com

${ }^{2}$ Faculdade deFarmácia, UniversidadeFederal de M inas Gerais
}

Abstract ThePharmaceutical Care concepts were analyzed from their origins in the United States and the later contributions which came from Spain and from the effort of sistematization by the World Health Organization to understand the processs that has been happening in Brasil. After theabandon of the communitarian pharmacy, the Brazilian pharmacists hope that this new model of practicing is the way to get back his/her social role. The philosophy which directs the Pharmaceutical Care, having the focus on patient, in our understanding, must support philosophical and conceptually the rebuilding of pharmaceutical practicing in Brazil in order to get back the lost relation between the pharmacist and patient at communitarian pharmacy.

Key words Pharmaceutical care, Community pharmacy, Pharmacist-patient relationship
Resumo Os conceitos de Atenção Farmacêutica foram analisados desde a sua origem, nos Estados Unidos, e as contribuições posteriores provenientes da Espanha e do esforço de sistematização pela Organização M undial da Saúde para compreender o processo que vem ocorrendo no Brasil. A pós o abandono das farmácias comunitárias, os farmacêuticos brasileiros esperam que esse novo modelo de prática seja um caminho para o resgate do seu papel social. A filosofia que orienta a Atenção Farmacêutica, o enfoquecentrado no paciente, em nosso entendimento, deve suportar fil losófica econceitualmente a reconstrução da prática farmacêutica no Brasil a fim de resgatar a relação farmacêutico-paciente nas farmácias comunitárias. Palavras-chave Atenção Farmacêutica, Farmácia comunitária, Relação farmacêutico-paciente 
Introdução

Este artigo se propõe a analisar a evolução do papel do farmacêutico na atenção à saúdee como, no Brasil, o desenvolvimento da Atenção Farmacêutica pode contribuir para a melhoria da atuação do profissional nas farmácias comunitárias, valorizando, consequentemente, o seu papel social.

Desde a sua proposição, muitas contribuições têm sido feitas no sentido de buscar o melhor entendimento eaplicação prática do conceito deAtenção Farmacêutica. Entre os conceitos e práticas da Atenção Farmacêutica desenvolvidos internacionalmente, devemos buscar as contribuições daqueles que são relevantes para o caso brasileiro. N este sentido, a análiseda origem do conceito nos Estados Unidos e das contribuições posteriores provenientes da Espanha e, por fim, do esforço de sistematização posto em marcha pela Organização Mundial da Saúde (OMS) são importantes para compreender o processo que vem ocorrendo no Brasil. Por meio de uma análise histórica e crítica dessas contribuições, contextualizando com o desenvolvimento da profissão farmacêutica no Brasil, este trabal ho pretende participar da construção deumaAten ção Farmacêutica voltada para a nossa realidade e necessidades.

\section{Uma breve história da farmácia no Brasil}

Desde o princípio, a profissão farmacêutica desenvolveu-se em um contexto de disputa com outros profissionais não diplomados, os práticos licenciados queatuavam nas boticas, os curandeiros, raizeiros e benzedores que prometiam a cura e tinham a confiança da população. Os boticários diplomados não podiam vir para a colônia, e a prática da farmácia continuaria baseada na experiência prática até a implantação da primeira escola de farmácia no Brasil, em 1832. As boticas possuíam uma importância social muito grande nas cidades brasileiras ${ }^{1-3}$, tendo se desenvolvido graças ao seu potencial comercial, embora o farmacêutico estabelecessecom seusclientesumare lação queia além da comercial. M as a industrialização do medicamento moderno iria influenciar a tomada de um novo rumo na profissão.

No início do século $X X$, os medicamentos eram produzidos de forma artesanal nas boticas e nas pequenas indústrias e possuíam valor terapêutico duvidoso dependente da percepção e do envolvimento ritual do usuário ${ }^{4}$. $M$ as descobertas terapêuticas importantes das décadas de 1930 e1940, relacionadas aos antimicrobianos, impul- sionaram a comunidade científica na busca por novas metodologias, iniciando o processo decrescimento do setor industrial ${ }^{5}$.

$\mathrm{N}$ asceu, assim, o medicamento moderno, racional e cientificamente explicado, conforme afirmam Perini eAcúrcio ${ }^{4}$, sendo seus efeitos demonstrados através de metodologias modernas de pesquisa. A produção artesanal de medicamentos foi gradualmente desaparecendo, sendo substituída pela industrial, feita em larga escala, e os produtos químico-sintéticos passaram a predominar nas indicações, modificando o papel do farmacêutico e da farmácia, que se transformava em pouco tempo em simples estabelecimento comercial.

Cabe lembrar que junto com o desenvolvimento da indústria farmacêutica emerge uma característica da sociedade moderna: o estímulo ao consumo de mercadorias. Pois, como afirma Sevalho ${ }^{5}$, "a produção crescente de tecnologias necessita de um mercado capaz de absorver a demanda de consumo quelheéincessantemente imposta e que, por isso, deve ser permanentemente ajustado ao poder da mídia e do instrumental simbólico da propaganda".

Esse novo medicamento surgido das transformações tecnológicas incorpora um sentido simbólico e, como afirma Sevalho ${ }^{5}$, podeser considerado um "objeto híbrido, uma mistura de bem de consumo einstrumento terapêutico". Em meio ao processo demedicalização da sociedade, o "negócio farmacêutico" tornou-se um disputado mercado em crescimento. As drogarias tornaram-se grandes parceiras das indústrias que se instalavam e dominavam o mercado farmacêutico brasileiro, distribuindo os seus produtos e estimulando o consumo dos medicamentos.

$\mathrm{Não}$ apenas no Brasil o farmacêutico manipulador perdeu espaço; as farmácias de manipulação não eram mais necessárias, pois os medicamentos já vinham prontos na forma de especialidades farmacêuticas. Os farmacêuticos não conseguiam inserção nas grandesindústrias enão tinham o que fazer nas drogarias, pois a comercialização dessas especialidades exigia menos conhecimento técnico. Além do mais, já em 1930, como aponta Giovanni ${ }^{6}$, com a al egação de que não existiam farmacêuticos suficientes para atender às necessidades do crescente número de farmácias no país, "a legislação passa a proporcionar a possibilidade" de seu funcionamento "sem a presença de farmacêuticos diplomados". Tinha início, assim, uma grande crise na profissão. No Brasil, houveuma migração de profissionais para campos como as análises clínicas, bromatológi- 
cas e toxicológicas, quenão têm a ver com o contexto do medicamento ${ }^{1}$

A farmácia transforma-se num estabelecimento de comércio de medicamentos. A regulamentação da atividade farmacêutica nas farmácias vigentedesde 1931 foi substituída pela Lei no 5.991, de 17 de dezembro de 1973, o que, na verdade, apenas reforçou o caráter comercial que 0 setor já vinha demonstrando, denominando, por exemplo, as atividades farmacêuticas de comércio farmacêutico?.

O profissional não consegue se inserir nessa prática e prefere outras áreas em que terá maior reconhecimento e prestígio. M as a responsabilidade técnica é uma exigência legal, e para cumpri-la os donos de drogarias contratam o farmacêutico apenas para "assinar" a responsabilidade técnica. 0 farmacêutico aceita essa situação, e a atividade estritamente farmacêutica é desmoralizada.

A indústria contribuiu para essa situação na medida em que passou a fornecer aos médicos, aos proprietários de farmácia e ao consumidor informações sobre as propriedades e indicações de uso dos produtos, na forma de bulas. 0 conhecimento do farmacêutico em relação aos me dicamentos é substituído pelas bulas, não havendo mais a necessidade do profissional para explicar os fatores envolvidos com a manutenção ea recuperação da saúde ${ }^{1}$. E numa época que exige temporalidades curtas porque "tempo é dinheiro", o atendimento tem que ser rápido e ágil para que se venda mais. $\mathrm{N}$ a medida em quea perspectiva social da compreensão da doença está ausente, ou depreciada, a antiga relação terapêutica farmacêutico-pacientenão tem razão de ser ${ }^{5}$.

$\mathrm{H}$ oje, o queencontramos na maioria das drogarias é a ausência ou inércia do farmacêutico e os balconistas e donos de drogarias atuando como prescritores, orientadores e conselheiros terapêuticos da população com o objetivo devender mais medicamentos.

O jovem Sistema Ú nico de Saúde (SUS) ainda vem sendo construído e, no seu contexto, no campo dos medicamentos e da assistência farmacêutica, os principais avançosocorreram, principalmente, após a aprovação da Política Nacional de M edicamentos (PNM), em outubro de 1998. Dentre as diretrizes e prioridades estabelecidas pela PNM , destacam-se a reorientação da assistência farmacêutica, a promoção do uso racional de medicamentos e a organização das atividades devigilância sanitária demedicamentos ${ }^{8,9}$.

No campo da vigilância sanitária, além das diretrizes estabelecidas pela PNM, várias medi- das de caráter normativo de medicamentos foram adotadas na década de 1990 e influenciaram a função do farmacêutico na farmácia comunitária. No final da década de 1990, ocorrências de falsificação e adulteração de medicamentos preocupavam a sociedade em relação à qualidade dos medicamentos comercializados, e isso contribuiu para a implantação de normas e exigências mais rigorosas por parte da vigilância sanitária no que diz respeito à produção, transporte e distribuição dos medicamentos no Brasil ${ }^{10}$.

A regulamentação dos medicamentos gené ricos instituiu normas para a prescrição e dispensação ${ }^{11}$. Ao farmacêutico coube a responsabilidade de fazer a troca, ou seja, substituir um medicamento dereferência pelo genérico, para o que ele deve estar presente na farmácia, e o usuário mais consciente dos seus direitos tem exigido o profissional capacitado para orientá-lo sobre a substituição e o uso do medicamento.

No Brasil, como já foi dito, a rápida expansão da industrialização do medicamento ea perda do papel tradicional dos farmacêuticos fizeram com que estes se afastassem do contato direto com o paciente. Em outros países, a história foi diferente. Os farmacêuticos estavam em busca da consolidação da sua importância como profissionais de saúde, e sua movimentação foi importante para a construção teórica e prática da Atenção Farmacêutica.

\section{A Atenção Farmacêutica}

Nos Estados Unidos, a perda do papel do farmacêutico nas farmácias após a industrialização foi solucionada no âmbito hospitalar através de uma nova disciplina que pretendia resgatar a participação do farmacêutico na equipe de saúde, a Farmácia Clínica.

0 código de ética da American Pharmacists Association (AphA) de 1952 estabelecia que os farmacêuticos não podiam discutir os efeitos terapêuticos dos medicamentos com os pacientes eque deveriam encaminhá-los ao médico ou dentista, caso houvesse alguma dúvida, acabando de vez com o poder que os farmacêuticos detinham sobre o uso dos medicamentos. Assim, os farmacêuticos comunitários se limitaram a comprar e vender medicamentos sem poder interferir na terapêutica. M as os farmacêuticos hospitalares realizavam outras atividades variadas, como a distribuição dentro da instituição e a participação na Comissão de Farmácia e Terapêutica. Dessa forma, eles resistiram à influência da indústria e dos médicos sobre o uso dos me- 
dicamentos e garantiram à farmácia uma voz na decisão terapêutica'².

Então começaram a surgir questionamentos sobre a função social da farmácia. Francke et al. ${ }^{13}$ realizaram um estudo sobre a prática farmacêutica em hospitais e afirmaram que a função de dispensação do farmacêutico é essencialmente uma prática superficial da profissão que não utiliza conhecimento ou habilidades suficientemente básicas para merecer o reconhecimento profissional.

Esses e outros questionamentos levaram Brodie e Benson ${ }^{14}$ a afirmar na década de 1960 que os farmacêuticos deveriam ser responsáveis pelo controle do uso de medicamentos. Surge, então, o movimento que resultou num modelo de prática farmacêutica, aplicado especialmente em hospitais, denominado Farmácia Clínica.

Hepler ${ }^{15}$ analisou a prática e a educação farmacêutica fazendo uma correlação com as três ondas socioeconômicas na história mundial (era agrária, industrial e da informação). Para ele, as três "ondas" da educação farmacêutica são a era do empirismo, da ciência e do cuidado ao paciente. 0 modelo que prevalecia até 1970 (era da ciência) formava cientistas e não profissionais. A ênfase nos processos mecânicos dos medicamentos acabou afastando o profissional da prática. A sociedade não necessitava de cientistas. Como nas farmácias não havia mais a atividade de manipulação, o farmacêutico passou a ocuparse com atividades gerenciais, e as farmácias tornaram-se canais dedistribuição da indústria. Para esse autor, o movimento clínico marca o início de uma nova era na educação e prática farmacêutica, a era do cuidado com o paciente. E foi importante para o resgate da função social da farmácia e profissionalização da prática nos hospitais e nas farmácias comunitárias.

A partir de então o conceito de Farmácia Clínica tem evoluído até incluir todas as atividades relacionadas ao uso racional eseguro do medicamento. Exemplo disso foi a modificação do código de ética profissional em 1969, quando os farmacêuticos foram encorajados a fornecer a cada paciente todas as suas habilidades e conhecimentos como um profissional de saúde essencial.

Porém, em 1990, Hepler e Strand ${ }^{16}$ publicaram um trabalho que influenciaria toda a profissão farmacêutica nos próximos anos. Embora reconhecessem a importância da farmácia clínica para a profissionalização da farmácia, esses autores consideram que algumas de suas definições situavam o medicamento em primeiro plano em detrimento do paciente. Então eles defendem que osfarmacêuticos, além de apoiar a concepção fun- cional da farmácia clínica, devem estar preparados para assumir a responsabilidade sanitária de prevenir a morbimortalidade relacionada com os medicamentos. Para isso, deverão modificar a sua prática. A profissão farmacêutica deve ser baseada em uma filosofia apropriada de prática e uma estrutura organizada que oriente a prática.

H epler eStrand ${ }^{16}$ defenderam a adoção deum enfoquecentrado no pacienteeo desenvolvimento deuma relação terapêutica na qual o pacientee o profissional trabalhem juntos para resolver os problemas relacionados aos medicamentos. Estes autores propuseram uma definição para a Atenção Farmacêutica quefoi amplamenteaceita pelos profissionais no mundo inteiro como ideia fundamental da profissão de farmácia: Provisão responsável do tratamento farmacológico com 0 propósito de alcançar resultados concretos que meIhorem a qualidadedevida do paciente. Estesresultados são: 1) a cura da doença, 2) a redução ou eliminação dos sintomas, 3) a interrupção ou retardamento do processo patológico, e 4) a prevenção de uma doença ou dos sintomas.

Em 1992, Cipolle et al. ${ }^{17}$ iniciaram um projeto de três anos para examinar de maneira crítica a relação existente entre a teoria emergente da Atenção Farmacêutica e a prática real, o M innesota Pharmaceutical Care Project. Esses autores desenvolveram um processo deresolução deproblemas que incorpora as responsabilidades do farmacêutico etentaram determinar se o conceito de Atenção Farmacêutica podia ser introduzido e desenvolvido na farmácia comunitária.

A partir dos resultados desse projeto, a definição original passou a ser considerada incompleta, e esses autores propuseram outra: prática na qual o profissional assume a responsabilidade pelas necessidades do paciente em relação aos medicamentos e um compromisso a respeito. Esta definição não foca os resultados como a anterior, mas reforça a necessidade de o profissional assumir a responsabilidade de resolver problemas farmacológicos complexos para que o exercício dessa nova prática profissional seja uma realidade.

Em uma publicação mais recente desses mesmos autores, o conceito de Atenção Farmacêutica foi novamente revisto. Reforçando o seu grande diferencial de propor uma prática centrada no paciente, os autores complementam a definição acrescentando: uma prática centrada no paciente na qual o profissional assume a responsabilidade pelas necessidades do paciente em relação aos medicamentos e um compromisso a respeito ${ }^{18}$.

Cipolleet al. ${ }^{17}$ consideram que a A ten ção Farmacêutica é uma prática como as demais da área 
de saúde. Possui uma filosofia, um processo de cuidado ao paciente e um sistema de gestão.

Uma filosofia do exercício profissional éuma série de valores que define as regras, funções, relações e responsabilidades do profissional. Por conseguinte, todos os profissionais que pretendem atuar consequentemente em um determinado exercício profissional devem refletir criticamente sobre a sua prática. A filosofia do exercício profissional éum componente prescritivo de uma prática e define o que se deve fazer. É um conjunto deideais, princípios, conceitos, valores e axiomas compartilhados por todos os profissionais equeservecomo referência para definir a natureza do seu exercício ${ }^{17}$.

Dessa forma, Cipolle et al. ${ }^{17}$ afirmam: A filosofia da atenção farmacêutica inclui diversos elementos. Começa com a "afirmação de uma necessidade social"; continua com um "enfoque centrado no paciente" para satisfazer esta necessidade; tem como elemento central a assistência a outra pessoa mediante 0 "desenvolvimento e manutenção de uma relação terapêutica", e finaliza com uma "descrição das responsabilidades concretas do profissional".

Para justificar sua posição e seus privilégios numa sociedade, todas as profissões devem satisfazer uma necessidade social específica. $\mathrm{Na}$ Atenção Farmacêutica, o profissional seencarrega de reduzir a morbidadee a mortalidade relacionadas com os medicamentos. 0 profissional satisfaz essa necessidade social atendendo às necessidades dos pacientes um a um. 0 farmacêutico é responsável por satisfazer a necessidade que tem a sociedade de receber um tratamento apropriado, efetivo, seguro e cômodo ${ }^{17}$.

Para isso, é necessário que o profissional da Atenção Farmacêutica utilizeem seu exercício um enfoque centrado no paciente. Isto significa que o paciente deveser considerado como pessoa em seu conjunto, com algumas necessidades de assistência gerais e outras específicas relacionadas com a medicação queconstituem a principal preocupação do profissional ${ }^{17}$. Segundo esses autores, o centro deinteresse da Atenção Farmacêutica passa do produto (medicamento) para a pessoa (paciente). Eles partem do conceito de que "os fármacos não tem doses, são as pessoas que recebem doses" 19 .

Como discutido por Ramalho de Oliveira ${ }^{20}$, o enfoquecentrado no pacienteéa experiência de atender ao chamado do "outro", o "outro" sendo o paciente que évulnerável e demanda atenção e responsabilidade profissional. Como discutido pela autora, para exercer a Atenção Farmacêuti- ca como prática centrada no paciente, além de competência técnica, o profissional deve deixar de ocupar o centro do seu universo cedendo este lugar ao paciente. 0 profissional deve estar sintonizado com o chamado do "outro", manter o interesse na subjetividade do paciente e contemplá-lo como uma pessoa única. Apesar de aparentemente básicas, as ideias de consideração do indivíduo e responsabilização por decisões terapêuticas são conceitos complexos e ainda não vivenciados pela maioria dos farmacêuticos no seu dia a dia de prática profissional. Principalmente quando consideramos o caso brasileiro, em que o farmacêutico historicamente afastouse do público que utiliza medicamentos.

O cuidado ou assistência ao paciente no contexto da atenção farmacêutica requer uma relação que promova a participação do paciente no processo terapêutico. Conforme dito por H epler e Strand ${ }^{16}$, a atenção farmacêutica se baseia em um acordo entre o paciente, que aceita conceder autoridade ao profissional, e o profissional, que garante ao paciente competência e compromisso. N esta perspectiva, estabelece-se uma relação de reciprocidade de responsabilidades do profissional e do paciente baseada no diálogo, na confiança, respeito, sinceridadee autenticidade, com a finalidade específica desatisfazer as necessidades de uma assistência sanitária devidamente contextualizada na complexidade social.

Além da filosofia que orienta a prática, um dos grandes diferenciais da Atenção Farmacêutica proposta por esses autores é o processo de cuidado ao paciente. Eles defendem que só deve existir um único processo de cuidado que deve ser padrão em qualquer lugar para qualquer farmacêutico, assim como são os processos para a atenção médica, para a atenção odontológica e para os cuidados de enfermagem. Trata-se de um processo de solução de problemas lógico, sistemático e global quepermiteao profissional prestar um serviço de qualidade, completo e uniforme ao paciente. São várias as vantagens citadas para se adotar um processo comum de assistência, dentreelas a capacidade de comunicação com os outros profissionais de saúde e outros farmacêuticos utilizando um vocabulário uniforme e, principalmente, a garantia de continuidade do serviço farmacêutico em todos os contextos de assistência sanitária: ambulatório, hospital e no domicílio ${ }^{17}$.

Conforme salientam os autores citados, filosofia, processo de cuidado e gestão da prática são três componentes essenciais de qualquer prática profissional. Porém, eles não são considera- 
dos para as práticas farmacêuticas atuais nas farmácias comunitárias. 0 farmacêutico, como já dito, perdeu seu espaço e reconhecimento nas farmácias comunitárias quando estas se tornaram simples estabelecimentos comerciais. Sua função como cuidador da saúde dos usuários de medicamentos não tinha mais valor, e a dispensação de medicamentos tornou-se um ato comercial. $\mathrm{Na}$ verdade, o farmacêutico ficou sem saber o que fazer nas drogarias, pois a sua filosofia não é adequada à característica do estabelecimento, e o processo de cuidado foi modificado a partir do advento das especialidades farmacêuticas com bulas.

Por isso, Cipolle et al. ${ }^{17}$ consideram que as funções tradicionais de dispensação não serão mais a razão de ser do farmacêutico, pois essa é uma atividade essencialmente técnica que pode ser realizada por auxiliares bem treinados. 0 farmacêutico deve assumir novas funções e responsabilidades centradastotalmenteno pacientecom a finalidade de resolver problemas farmacológicos complexos. Eles, então, defendem a preparação de um novo profissional que se encarregará de uma nova prática profissional na qual oferecerá um novo serviço, a Atenção Farmacêutica ${ }^{17}$.

Esse novo modelo de prática ganhou campo nas discussões internacionais sobre o papel do farmacêutico. Então surgiram questionamentos sobre os fundamentos da Atenção Farmacêutica. Seria esse o único modelo de prática profissional? E as outras atividades do farmacêutico, como a dispensação, a informação sobre medicamentos, a indicação, educação em saúde?

Os próprios autores desse modelo, na segunda edição do seu livro ${ }^{18}$, reafirmam quea Atenção Farmacêutica é uma nova prática profissional. Porém, agora eles assumem que esta prática não pretende substituir o farmacêutico dispensador.

Muitos trabalhos foram desenvolvidos em diferentes localidades tentando propor uma solução a esses anseios. Um exemplo disso são os trabal hos de Holland e $\mathrm{N}$ immo ${ }^{21-25}$, pesquisadores australianos que defendem o não esquecimento das atividades clínicas dos farmacêuticos, propondo um novo modelo de Atenção Farmacêutica Integral queinclui cinco outras atividades farmacêuticas como submodelos.

\section{As contribuições da Espanha}

$\mathrm{Na}$ Espanha foi produzido um consenso em 2001, que incluiu as outras atividades voltadas para o paciente no conceito de Atenção Farmacêutica: A participação ativa do farmacêutico na assistência ao pacientena dispensação e seguimento do tratamento farmacoterapêutico, cooperando com o médico e outros profissionais de saúde, a fim de conseguir resultados que mel horem a qualidade de vida dos pacientes. Também prevêa participação do farmacêutico em atividades de promoção à saúde e prevenção de doenças ${ }^{26}$.

$\mathrm{Na}$ perspectiva espanhola, as atividades farmacêuticas orientadas para o paciente, como a dispensação, a indicação de medicamentos que não necessitam dereceita médica, educação sanitária, farmacovigilância, o seguimento farmacoterapêutico e todas aquelas que se relacionam com o uso racional de medicamentos foram incluídas no modelo de prática profissional denominado Atenção Farmacêutica. Com isto pretende-se potencializar as atividades assistenciais do farmacêutico como profissional de saúde ${ }^{26}$.

0 que a perspectiva espanhola define como Seguimento Farmacoterapêutico assemelha-seao conceito deAtenção Farmacêutica elaborado pelo grupo de Minnesota. O Consenso Espanhol expressa que: Seguimento farmacoterapêutico individualizado é a prática profissional na qual o farmacêutico se responsabiliza pelas necessidades do paciente relacionadas com os medicamentos mediante a detecção, prevenção e resolução de problemas relacionados com a medicação (PRM ), deforma continuada, sistematizada e documentada, em colaboração com o próprio paciente e com os demais profissionais do sistema de saúde, com o fim de alcançar resultados concretos que melhorem a qualidade de vida do paciente ${ }^{26}$.

Assim, a perspectiva espanhola édiferentedo grupo de Minnesota. Enquanto os americanos consideram a Atenção Farmacêutica uma prática única e diferente das demais atividades realizadas pelo farmacêutico e com um processo racional de tomada de decisão, os espanhóis reconhecem as atividades farmacêuticas tradicionais como a dispensação como parte deste novo modelo de prática, pois em todas elas o farmacêutico está assumindo a responsabilidade pela redução da morbimortalidade relacionada aos medicamentos. No entanto, os mesmos pilares filosóficos sustentam as duas perspectivas.

\section{As contribuiç̧ões da Organização Mundial da Saúde}

A Organização Mundial daSaúde(OM S) vem produzindo uma série de recomendações internacionais relacionadas aos medicamentos e ao papel do farmacêutico no sistema de atenção à saúde e, dessa forma, tem contribuído para a 
construção conceitual da Atenção Farmacêutica. Deve-se destacar dois documentos muito importantes para o contexto dessa discussão: os informes produzidos pelas reuniões de Nova Délhi, em 1988, eTóquio, em 1993.

Em N ova Délhi, ressaltou-se a importância do farmacêutico no sistema de atenção à saúde, descrevendo as suas funções na equipe de saúde, especialmente em relação aos seus conhecimentos sobre o manejo e as propriedades dos medicamentos. 0 farmacêutico, segundo o grupo reunido, deve atuar como fonte de informação sobre os medicamentos para a equipe e os pacientes, sendo o responsável pelo controle do uso racional e seguro do medicamento. Ou seja, a OMS adota as ideias da farmácia clínica como diretrizes para a profissão farmacêutica ${ }^{27}$.

Após a divulgação do trabalho de Hepler e Strand ${ }^{16}$ sobre a Atenção Farmacêutica, a OM S promove um novo encontro para rever o papel do farmacêutico em 1993. 0 documento produzido nessa reunião éconhecido como Declaração de Tóquio e adota um conceito de Atenção Farmacêutica reforçando a reorientação do produto para o paciente: U m conceito de prática profissional na qual o paciente éo principal beneficiário das ações do farmacêutico. A Atenção Farmacêutica é o compêndio das atitudes, dos comportamentos, dos compromissos, das inquietudes, dos valores éticos, das funções, dos conhecimentos, das responsabilidades e das destrezas do farmacêutico na prestação da farmacoterapia, com o objetivo de alcançar resultados terapêuticos definidos na saúde ena qualidade de vida do paciente ${ }^{28}$.

Além disso, a O M S reconhece o farmacêutico como dispensador de atenção sanitária, ressaltando que os benefícios da Atenção Farmacêutica devem ser direcionados ao paciente e à comunidade através de ações de promoção da saúde e prevenção de doenças. 0 grupo reunido em Tóquio, ao destacar os elementos da aten ção farmacêutica ao paciente, inclui diversas outras atividadesfarmacêuticas ao processo de cuidado do paciente, como a dispensação e a indicação farmacêutica. Complementando, a O M S recomenda que a Atenção Farmacêutica seja uma atitude profissional a que todo farmacêutico deve visar ${ }^{28}$.

A Organização M undial da Saúde, juntamente com organizações profissionais farmacêuticas, vem exercendo uma influência muito importante na definição do papel do farmacêutico e de políticas farmacêuticas em vários países. Esta influência está baseada, principalmente, na recomendação de que todo o farmacêutico deve adotar o conceito de atenção farmacêutica na sua prática profissional. Como as outras recomendações levam em conta as atividades tradicionais do farmacêutico relacionadas ao paciente eà comuni dade, a adoção do conceito deAtenção Farmacêutica estaria no plano das ideias, do comportamento e das atitudes dos farmacêuticos na sua prática atual para melhorar a assistência ao paciente e à comunidade no que se refere à utilização de medicamentos.

Assim, a perspectiva da OMS é diferente da posição do grupo de Minnesota, pelo menos no que diz respeito à incorporação de outras atividades farmacêuticas no âmbito da Atenção. A parentemente, a proposta da OM S aproxima-se mais da concepção espanhola e é assim que tem influenciado a posição brasileira.

\section{O caso do Brasil}

No Brasil, as atividades do farmacêutico estão inseridas na Assistência Farmacêutica, um conceito cuja construção teve início no final dos anos 1980 envolvendo todas as atividades relacionadas ao medicamento desde a pesquisa e produção até a dispensação de forma cíclica e integrada.

Dez anos depoiséinstituída a Política N acional de Medicamentos (PNM), que reorienta 0 conceito de assistência farmacêutica com o propósito de garantir a segurança, a eficácia ea qualidade dos medicamentos, bem como a promoção do uso racional e o acesso da população aos medicamentos considerados essenciais, respondendo à necessidade, discutida internacional mente há anos, de racionalizar o uso de medicamentos e aumentar o acesso e reafirmando que os farmacêuticos deveriam assumir essa responsabilidade. Assim, a Assistência Farmacêutica é conceituada como um grupo de atividades relacionadas com o medicamento, destinadas a apoiar as ações de saúdedemandadas por uma comunidade. Envolve 0 abastecimento de medicamentos em todas e em cada uma de suas etapas constitutivas, a conservação e controle de qualidade, a segurança e a eficácia terapêutica dos medicamentos, 0 acompanhamento e avaliação da utilização, a obtenção e a difusão de informação sobre medicamentos e a educação permanente dos profissionais de saúde, do paciente e da comunidade para assegurar o uso racional de medicamentos ${ }^{8}$.

Porém, as atividades continuavam centradas no medicamento, o queera evidenciado especialmente pela não adoção do novo modelo de prática, a Atenção Farmacêutica, que já havia sido recomendado pela O M S em 1993, na D eclaração de Tóquio. 
Em relação ao conceito de dispensação também se observa um avanço, pois diferentemente do conceito proposto pela legislação sanitária, o aspecto comercial da atividade é excluído. Ao deixar claro que o farmacêutico éresponsável não só pelo fornecimento do medicamento como também pela orientação para o seu uso adequado, o conceito de dispensação adquire um caráter mais técnico. Dispensação éo ato profissional farmacêutico de proporcionar um ou mais medicamentos a um paciente, geralmente como resposta à apresentação de uma receita elaborada por um profissional autorizado. N este ato o farmacêutico informa e orienta o paciente sobre o uso adequado do medicamento ${ }^{8}$.

M esmo com a definição de conceitos relacionados à prática farmacêutica na PN M , o ConseIho Federal de Farmácia (CFF) publica uma re solução, em 2001, queestabelece as Boas Práticas em Farmácia propondo outro conceito de assistência farmacêutica, que inclui a preocupação demonstrada nos informes da OMS sobre a assistência integral à saúde, mas não especifica as atividades farmacêuticas ${ }^{29}$. Da mesma forma como na PNM , ainda não há a adoção do conceito deAtenção Farmacêutica, embora seja possível notar alguns avanços operacionais no estabelecimento de requisitos para a prática farmacêutica, especialmenteem farmácias comunitárias, que se assemel ham aos procedimentos da farmácia clínica e da Atenção Farmacêutica.

Já em relação ao conceito de dispensação não há avanço na resolução do CFF, e em relação à PNM há um retrocesso, mantendo o caráter comercial da atividade: ato do farmacêutico de orientação e fornecimento ao usuário de medicamentos, insumos farmacêuticos e correlatos, a título remunerado ou não29.

A Farmácia Clínica chegou ao Brasil muito timidamente, e apenas alguns hospitais chegaram a colocar em prática esse modelo. 0 primeiro serviço foi implantado em N atal, no Rio Grande do Norte, em $1979^{30}$.

Em relação à Atenção Farmacêutica, o processo brasileiro também se iniciou tardiamentee com confusão conceitual. 0 próprio CFF, em publicações nos anos de 1996 e 1997, apresenta o conceito deAtenção Farmacêutica como sendo o de assistência farmacêutica ${ }^{31,32}$.

Produziram-se, então, no Brasil, diferentes interpretações sobre os conceitos de Atenção e Assistência Farmacêutica, sobre a operacionalização e inter-relação deles e sobre os diferentes entendimentos do papel do farmacêutico e do objeto de sua prática no país.
Então, em 2002, um grupo de profissionais coordenado pela Organização Pan-Americana da Saúde (O pas) reuniu-se com o objetivo de discutir a uniformização de termos econceitos e paraa promoção da Atenção Farmacêutica no país. Os resultados desses trabal hos foram publicados em um relatório que posteriormente deu origem a uma Proposta de Consenso Brasileiro de Atenção Farmacêutica. $O$ conceito deAssistência Farmacêutica foi revisado, e no entendimento dos participantes é diferente de Atenção Farmacêutica, tem características multiprofissionais e engloba todas as atividades relacionadas ao abastecimento e utilização de medicamentos ${ }^{33,34}$.

A Atenção Farmacêutica refere-se às atividades específicas do farmacêutico no cuidado do paciente ou usuário do medicamento: É um modelo de prática farmacêutica, desenvolvida no contexto da Assistência Farmacêutica. Compreende atitudes, valores éticos, comportamentos, habilidades, compromissos e co-responsabilidades na prevenção de doenças, promoção e recuperação da saúde, de forma integrada à equipe de saúde. É a interação direta do farmacêutico com o usuário, visando uma farmacoterapia racional e a obtenção de resultados definidose mensuráveis, voltados para a melhoria da qualidade devida. Esta interação também deve envolver as concepções dos seus sujeitos, respeitadas as suas especificidades biopsicossociais, sob a ótica da integralidade das ações de saúde ${ }^{34}$.

Este conceito deAtenção Farmacêuticaémuito mais amplo do queos citados anteriormenteneste trabalho; envolve termos que necessitam de outras definições ou explicações e não é objetivo nem preciso na definição de responsabilidades específicas do farmacêutico.

0 conceito não avança em relação ao original de H epler e Strand ${ }^{16}$, complementado por CipoIleet al. ${ }^{16}$, queé mais objetivo e estabelece de forma clara a responsabilidade específica do farmacêutico que pratica Aten ção Farmacêutica: "definição das necessidades farmacoterápicas do paciente e o compromisso deresolvê-las"; pois "atitudes, valores éticos, comportamentos, habilidades, compromissos e co-responsabilidades" são qualidades inespecíficas equeestão presentes em qualquer prática profissional. Segundo esse conceito, elas estão relacionadas a atividades que são realizadas por outros profissionais de saúde em conjunto com o farmacêutico: "ações de prevenção de doenças, promoção erecuperação da saúde, de forma integrada à equipe de saúde". No entanto, ao incluir essas atividades como responsabilidade do farmacêutico, o conceito está seguindo a tendência ideológica do sistema de saú- 
de brasileiro e seus princípios doutrinários de universalidade, equidade eintegralidade. A perspectiva da integralidade das ações de saúde também pode ser traduzida na necessidade de as atividades profissionais envolverem "as concepções dos seus sujeitos, respeitadas as suas especificidades biopsicossociais", considerando o indivíduo como um ser único.

Como ocorreu no consenso espanhol, as atividadesfarmacêuticas relacionadas ao cuidado do paciente foram consideradas componentes da prática profissional para o exercício da Atenção Farmacêutica. Os denominados macrocomponentes da prática da Atenção Farmacêutica são: educação em saúde, incluindo promoção do uso racional de medicamentos; orientação farmacêutica; dispensação; atendimento farmacêutico; acompanhamento/seguimento farmacoterapêutico; registro sistemático das atividades, mensuração e avaliação dos resultados ${ }^{34}$. O u seja, além de incluir atividades estritamente farmacêuticas, ela adiciona atividades multiprofissionais e procedimentos relacionados a várias atividades. $\mathrm{A}$ confusão torna-se maior ao utilizar termos pouco conhecidos pelos profissionais, como atendimento farmacêutico, cujo conceito não explica claramente do que se trata, podendo ser interpretado de diversas formas. Além disso, alguns termos como orientação farmacêutica não são conceituados. Pode-se considerar tanto um serviço farmacêutico (indicação de medicamentos que não necessitam de prescrição médica ou condutas não farmacológicas para tratamento de problemas simples) quanto qualquer intervenção realizada pelo farmacêutico baseada em fornecimento de informações e/ou esclarecimento de dúvidas proveniente de diversas situações na farmácia.

No entanto, considerando a missão da prática farmacêutica definida pela OM S, as atividades de educação em saúde, especialmente relacionadas ao uso correto de medicamentos, a dispensação ea orientação (entendida como indicação farmacêutica) podem interferir de maneira significativa no uso adequado de medicamentos pelas pessoas e pela sociedade e, por conseqüência, na prevenção e resolução de problemas relacionados aos medicamentos.

Essa realidade é ilustrada pelos resultados de um estudo de avaliação deum serviço deAtenção Farmacêutica em Belo Horizonte (M G), realizado por Nascimento ${ }^{35}$. 0 estudo demonstrou que grande número de problemas identificados durante 0 acompanhamento farmacoterapêutico é relativo à efetividade dos medicamentos. Segundo a autora, tais problemas podem ser resolvi- dos, em princípio, pela introdução de medidas não farmacológicas, implantação de estratégias para aumentar a adesão aos tratamentos farmacológicos ou não, educação sobre o uso correto do medicamento, educação em saúde, indicação demedicamentos de venda livre, além do encaminhamento para outro profissional de saúde.

0 fato de o Consenso Brasileiro de Atenção Farmacêutica propor um conceito amplo deAtenção Farmacêutica caracteriza-o como um documento teórico redirecionador das práticas farmacêuticas. Ao englobar as ações de saúde concordantes com os princípios do SUS e incluir outras atividades do farmacêutico relacionadas ao paciente, o documento está considerando a realidade brasileira da profissão farmacêutica e das farmácias comunitárias no país. No Brasil, como já discutido, há uma necessidade histórica de resgate da relação farmacêutico-paciente nas farmácias e de valorização profissional.

M esmo elas sendo consideradas estabelecimentos comerciais, acreditamos que as farmácias comunitárias devem manter alguns serviços farmacêuticos essenciais para garantir queo usuário de medicamentos receba medicamentos de qualidade e sejam orientados adequadamente a respeito do uso correto de medicamentos e cuidados com a saúde.

Desde a elaboração da Proposta de Consenso Brasileiro de Atenção Farmacêutica, vários eventos já foram realizados com o intuito decontinuar a divulgação desse novo modelo de prática no Brasil. Nos últimos anos, o tema vem sendo discutido em vários congressos elistas de discussão, tanto no âmbito nacional como no internacional. Muitos profissionais brasileiros estão envolvidos nesse movimento em busca da consolidação dessa prática farmacêutica.

M esmo com todas as diferenças em conceitose definições discutidas por vários autores e em váriospaíses, a fil losofia da Atenção Farmacêutica permanececonsensual, principalmenteo enfoquecentrado no paciente. Esta é a diferença fundamental em relação aos modelos de prática profissional em que o foco era o produto, o medicamento.

Acreditamos que a adoção dafilosofia de prática proposta pela Atenção Farmacêutica pode ser um caminho para o resgate da dignidade profissional, da relação farmacêutico-paciente e da reinserção do farmacêutico na equipe de saúde. Os farmacêuticos devem, portanto, entender, adotar e praticar a filosofia da Atenção Farmacêutica na sua prática diária abandonando a posição de meros entregadores de medicamentos, contribuindo assim, de fato, para a melhoria da 
saúde e da qualidade de vida dos usuários de medicamentos, bem como para sua utilização mais racional no Brasil.

\section{Consideraçõesfinais}

Finalmente, após essa análise, podemos verificar que a Atenção Farmacêutica, segundo o grupo de M innesota, é uma nova prática profissional, que exige um novo profissional, e somente dessa forma é que os farmacêuticos poderão exercer a sua função social traduzida na responsabilidade e no compromisso com a resolução de problemas farmacológicos complexos.

$\mathrm{Na}$ Espanha, o entendimento é de queas práticas antigas devem ser reestruturadas de acordo com a nova filosofia da Atenção Farmacêutica, para quea responsabilidade do farmacêutico com a assistência ao paciente e com a promoção do uso racional de medicamentos seja resgatada.

A OMS, em seus documentos sobre a função do farmacêutico, também segue essa tendência, ampliando o conceito de Atenção Farmacêutica e estabelecendo que essa é a missão da prática farmacêutica.

No Brasil, o histórico da profissão farmacêutica evidencia a influência de interesses comerciais no abandono da farmácia por parte do profissional ena perda da relação farmacêutico-paciente. A Atenção Farmacêutica como proposta no Consenso Brasileiro, embora defina conceitos pouco específicos eainda confusos, incorpora elementos característicos do sistema de saúde brasileiro que contribuem para o redirecionamento da prática farmacêutica brasileira.

M as, em nosso entendimento, éa filosofia da Atenção Farmacêutica, aplicada em todas as atividades dos farmacêuticos relacionadas ao paciente, que dará suporte para a recuperação da função do farmacêutico nas farmácias comunitárias e reconstrução da relação farmacêutico- paciente. Quando o farmacêutico assume a responsabilidade pelo cuidado do paciente, direcionando as suas ações pelo estabelecimento de uma relação terapêutica de respeito e confiança, reafirma o seu papel na equipe de saúde e revela o seu valor social.

Em nosso entendimento, o farmacêutico deve ser de fato um dos responsáveis pela terapêutica enão um mero subordinado à autoridade médica, e somente nessa posição ativa poderá atuar na promoção do uso racional do medicamento e transformar a farmácia em estabelecimento de saúde.

Porém, al gumas deficiências precisam ser corrigidas, por exemplo, em relação à formação e capacitação do farmacêutico, que deve ser criticamente voltada para um novo perfil profissional. 0 farmacêutico precisa ser formado para ser um profissional da saúde que pense criticamente a sua prática, e não mais um técnico em medicamentos.

Finalmente, acreditamos que é necessário continuar o trabalho iniciado na elaboração do Consenso Brasileiro para divulgar e promover esse novo conceito de prática, de forma que os farmacêuticos brasileiros possam compreender os seus princípiosfilosóficos e aplicar na sua prática diária. E mais ainda, precisamos rever conceitos e práticas farmacêuticas incorporando essa nova filosofia em todas as atividades farmacêuticas direcionadas aos pacientes, pois todas contribuem para a melhoria das condições de saúde e de utilização dos medicamentos pela população brasileira.

Porém, incorporar essa nova filosofia e mudar de atitude não garante uma mudança na realidade dos serviços farmacêuticos prestados em farmácias comunitárias se não vier acompanhada de uma reorganização do processo de cada um dos serviços. Somente assim o farmacêutico saberá como aplicar essa filosofia em benefício do paciente. 


\section{Colaboradores}

D Angonesi foi responsável pela concepção, pesquisa e redação final do artigo; G Sevalho foi 0 orientador da pesquisa e também responsável pela elaboração do artigo.

\section{Referências}

1. Santos MR. Do boticário ao bioquímico: as transformações ocorridas com a profissão farmacêutica no Brasil [dissertação]. Rio de Janeiro: Escola Nacional de Saúde Pública Sergio Arouca; 1993.

2. Silva BC. Direito farmacêutico. São Paulo: Fundo Editorial BYK Procieny; 1983.

3. Souza MA, Dupim JAA, Chaves JF, Steffens MH, Faria ACCF, Cardoso JSM, Ferreira GM, Santos JCM. A pontamentos para a história da farmácia em $M$ inas Gerais: práticas farmacêuticas e construção do saber farmacêutico. In: Soares A, Barbosa MV, organizadores. Centro Universitário N ewton Paiva: Iniciação Científica 2002-2003. Belo Horizonte: Centro Universitário Newton Paiva; 2003. p. 40-63.

4. Perini E, Acúrcio, FA. Farmacoepidemiologia. In: Gomes MJVM, Reis AM M, organizadores. Ciências farmacêuticas: uma abordagem em farmácia hospitalar. Belo Horizonte: Atheneu; 2001. p. 85-107.

5. Sevalho G. O medicamento percebido como objeto híbrido: uma visão do uso racional. In: Acúrcio FA, organizador. M edicamentos e Assistência Farmacêutica. Belo Horizonte: Coopmed; 2001. p. 1-8.

6. Giovanni G. A questão dos remédios no Brasil: produção e consumo. São Paulo: Polis; 1980.

7. Cunha BCA. M edicamentos: fator de saúde? São PauIo: Artpress; 1981.

8. Brasil. M inistério da Saúde. Secretaria de Políticas de Saúde. Portaria n 3.916, de 30 de outubro de 1998. Aprova a Política Nacional de Medicamentos. Diário O ficial da União 1998; 10 nov. Seção 1, p. 18-22.

9. Acúrcio FA. Política de medicamentos e Assistência Farmacêutica no Sistema Ú nico de Saúde. In: Acúrcio FA, organizador. M edicamentos e Assistência Farmacêutica. Belo Horizonte: Coopmed; 2003. p. 9-30.

10. M elo M GM. A regulamentação sanitária e a sua influência na definição do cenário farmacêutico do país. In: Acúrcio FA, organizador. M edicamentos e Assistência Farmacêutica. Belo Horizonte: Coopmed; 2003. p. 61-82.

11. Brasil. Congresso Nacional. Lei n० 9.787, de 10 de fevereiro de 1999. Altera a Lei $n^{\circ} 6.360$, de 23 de setembro de 1976, que dispõe sobre a vigilância sanitária, estabelece o medicamento genérico, dispõe sobre a utilização de nomes genéricos em produtos farmacêuticos e dá outras providências [acessado 2002 out 30]. Disponível em: http://www.cff. org.br/userfiles/file/leis/9787.pdf

12. Higby GJ. The continuing evolution of American pharmacy practice, 1952-2002. J Am Pharm Assoc 2002; 42(1):12-15.

13. Francke DE, Latiolais CJ, Francke GN, Ho NF. Mirror to the hospital pharmacy. Am Soc Hosp Pharm 1964; apud Hepler CD. The third wave in pharmaceutical education: the clinical movement. Am J Pharm Educ 1987; 51:369-384.

14. Brodie DC, Benson RA. The evolution of the clinical pharmacy concept. Drug Intellig Clin Pharm 1976; 10:507; apud Higby GJJ. The continuing evoIution of American pharmacy practice, 1952-2002. Am Pharm Assoc 2002; 42(1):12-15. 
15. Hepler CD. The third wave in pharmaceutical education: the clinical movement. Am J Pharm Educ 1987; 51:369-384.

16. Hepler CD, Strand LM. Opportunities and responsibilities in pharmaceutical care. Am J Hosp Pharm 1990; 47:533-543.

17. Cipolle RJ, Strand LM, Morley PC. El ejercicio de la atención farmacéutica. Madrid: M cGraw-Hill Interamericana; 2000. p. 1-35.

18. Cipolle RJ, Strand LM, M orley PC. Pharmaceutical care practice: the clinician's guide. N ew York: M cGrawHill; 2004. p. 2-4.

19. Cipolle RJ. Drugs don't have doses... People have doses. Drug Intel Clin Pharm 1986; 20:881-882; apud Cipolle RJ, Strand LM, Morley PC. El ejercicio de la Atención Farmacéutica. Madrid: M cGraw-Hill Interamericana; 2000. p. 1-35.

20. Ramalho de Oliveira D. Pharmaceutical Care uncovered: an ethnographic study of pharmaceutical care [tese]. M innesota: University of M innesota; 2003.

21. Holland RH, Nimmo CM. Transitions in pharmacy practice part 1: beyond pharmaceutical care. Am J Health-Syst Pharm 1999; 56(17):1758-1764.

22. Holland $\mathrm{RH}, \mathrm{Nimmo} \mathrm{CM}$. Transitions in pharmacy practice, part 2: who does what and why. Am J Health-Syst Pharm 1999; 56(19):1981-1987.

23. Holland RH, Nimmo CM. Transitions in pharmacy practice, part 3: effecting change-the-three-ring cirCus. Am J H ealth-Syst Pharm 1999; 56(21):2235-2241.

24. Holland RH, Nimmo CM. Transitions in pharmacy practice, part 4: can a leopard change its spots? Am J H ealth-Syst Pharm 1999; 56(23):2458-2462.

25. Holland RH, Nimmo CM. Transitions in pharmacy practice, part 5: walking the tightrope of change. Am J H ealth-Syst Pharm 2000; 57(1):64-72.

26. España. M inistério de Sanidad y Consumo. Consenso sobre Atención Farmacéutica. M adrid: M inistério de Sanidad y Consumo; 2001.

27. Organización M undial de la Salud (OMS). El papel del farmacéutico en la atención a la salud: informe de un grupo de consulta de la OMS, Nueva Delhi, India, 13-16 dic, 1988. Genebra: WH O; 1990.
28. Organización Pan-Americana de la Salud/Organización Mundial de la Salud (Opas/OMS). El papel del farmacéutico en la atención a salud: informe de la reunión de la OMS, Tokio, Japon, 31 ago al 3 sep 1993. Buenas Prácticas de Farmácia: N ormas de Calidad de los Servicios Farmacéuticos. La Declaración de Tokio - Federación Internacional Farmacéutica. Washington: PAHO; 1995.

29. Conselho Federal de Farmácia (CFF). Resolução $n^{\circ}$ 357. A prova o regulamento técnico das Boas Práticas de Farmácia. Pharm Bras 2001; 25:5-12.

30. Palhano TJ. Farmácia dínica, aconselhamento ao paciente, RAM . Belo Horizonte: Faculdade de Farmácia, Universidade Federal de M inas Gerais; 2002. (M aterial de apoio do Curso de Especialização em Saúde Pública, área de concentração medicamentos).

31. Conselho Federal de Farmácia (CFF). O papel do farmacêutico no sistema de assistência à saúde. Pharm Bras 1996; 3:37-40.

32. Conselho Federal de Farmácia (CFF). Farmácia clínica é tema de congresso latino-americano. Pharm Bras 1997; 6:36.

33. Organização Pan-Americana da Saúde (O pas). Atenção Farmacêutica no Brasil trilhando caminhos: relatório 2001-2002. Brasília: O pas; 2002.

34. Organização Pan-Americana da Saúde (O pas). Consenso Brasileiro de Atenção Farmacêutica: proposta. Brasília: O pas; 2002.

35. Nascimento YA. Avaliação de resultados de um serviço de atenção farmacêutica em Belo H orizonte [dissertação]. Belo Horizonte: Faculdade de Farmácia, Universidade Federal de M inas Gerais; 2004.

Artigo apresentado em 07/07/2008

Aprovado em 10/09/2008

Versão final apresentada em 30/10/2008 